\title{
Processes for the Self-assembly of Micro Parts
}

\author{
Matthias Burgard ${ }^{1}$, Norbert Schläfli ${ }^{2}$, and Uwe Mai ${ }^{1}$ \\ ${ }^{1}$ Fraunhofer Institute for Manufacturing Engineering and Automation, \\ Nobelstr. 12, 70569 Stuttgart, Germany \\ \{matthias.burgard, uwe.mai\} @ipa.fraunhofer.de \\ ${ }^{2}$ Norbert SchläfliMaschinen, Mühlethalstrasse 69, 4800 Zofingen, Switzerland \\ ns@nsmz.com
}

\begin{abstract}
The following approach of a fluidic based self-assembly process uses the surface forces for a precise handling and positioning of small devices for a roll-to-roll manufacturing. The surface, on which the device should be positioned, will be functionalized with hydrophile and hydrophobic areas. Thus water droplets can be caught on the hydrophile areas. The devices to be positioned will be placed on these droplets and due to the surface tension moved to their final position. The droplets, a solution of ultrapure water and isopropanol, are evaporating residue-free within seconds [1].
\end{abstract}

Keywords: handling, automation, manufacturing, assembly, self-assembly, surface tension, fluid, roll-to-roll.

\section{Introduction}

The roll-to-roll manufacturing technology is used for a high throughput production and is widely-used in the printing industry. This technology is getting more and more important also for the electronic industry, especially for the manufacturing of organicand printed electronics and the manufacturing of microsystems [2]. The technology is based on a layer by layer approach to avoid serial processes, which are slowing down the production speed. Therefore, an integration of devices during the roll-to-roll manufacturing is not advisable nowadays. But the combination of conventional devices (e.g. semiconductor circuits, LEDs, sensors) with a roll-to-roll organic and printed electronics could take specific advantages of both technology platforms and could lead to new products with high functionalities [3]. Also the long lasting trend of miniaturization leads to increasing functionality but also to continuously shrinking devices. These small devices have to be automatically handled in assembly processes [4]. LEDs (light emitting diode) are reaching nowadays a feed size of $300 \mu \mathrm{m}$. The automatic handling and positioning of devices is getting more complex with its miniaturization. Surface forces play an increasing role and affect the common handling process [5]. These effects are occurring at device sizes of approx. $500 \mu \mathrm{m}$ and smaller [6]. 
The self-assembly process is using these surface forces for a precise handling and positioning of small devices and provides a high-throughput and cost efficient assembly for an inline integration of components in a roll-to-roll manufacturing.

Self-assembly processes are a known method for a highly parallel assembly of small parts [7], [8]. The described processes chain in [7] using the self-assembly effect have a sequential arrangement. The fluidic self-assembly for the integration of GaAsdevices that utilizes fluid transport and shape differentiation for placement and orientation requires well defined structures as well on the device as on the substrate [8]. For a roll-to-roll manufacturing a seamless processing has to be provided and to keep the manufacturing cost of devices as low as possible, their design restrictions should be minimized.

\section{The Overall Process}

The surface, on which the device should be positioned, is functionalized in hydrophobic and hydrophile areas. The hydrophile areas are surrounded by a hydrophobic surface and have the same shape and dimension as the device to be positioned. The hydrophilearea is wetted with ultrapure water and caught by the hydrophobic surroundings. The device is deposited on this droplet and due to surface tension aligned according the hydrophile shape.

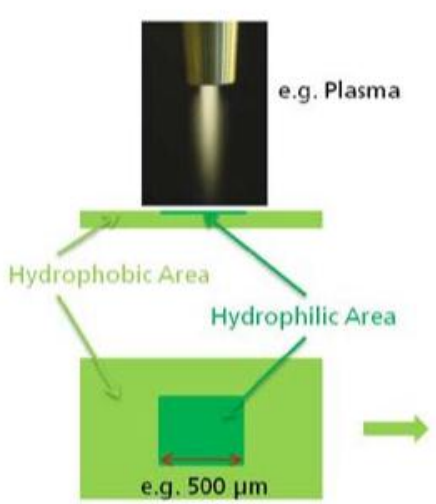

Surface Functionalization

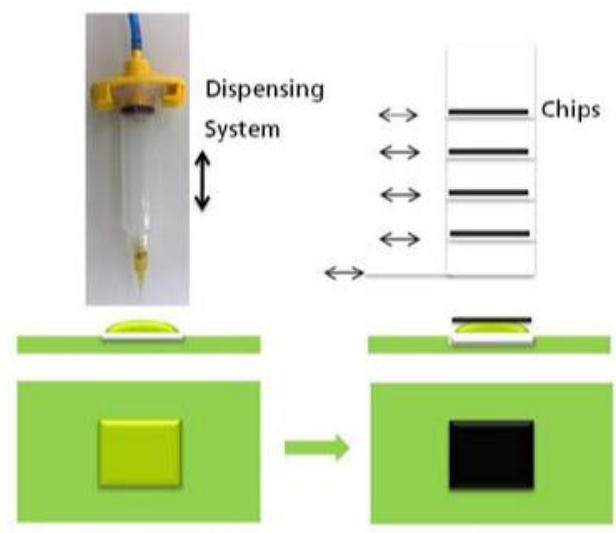

Chip deposition

Fig. 1. Schematic diagram of the process steps for the self-assembly of micro parts

\subsection{Surface Functionalization}

The requirements of the functionalized areas are:

- Sharp transition from hydrophobic to hydrophile characteristic

- Same shape and size of functionalized area and device e.g. 500 x $500 \mu \mathrm{m}$ 


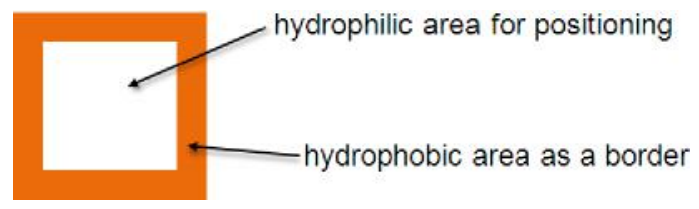

Fig. 2. Schematic of the functionalized area

The surface functionalization can be realized by different methods. Investigations have shown that atmospheric plasma and the technology for the manufacturing of offset-printing plates can be used to realize the required functionalized areas with an edge length below $500 \mu \mathrm{m}$.

For the atmospheric plasma functionalization a polymer is used as substrate. An SMD (Surface Mounted Device)-mask with apertures of e.g. $500 \mu \mathrm{m}$ is placed on the polymer. The plasma nozzle with exhausting atmospheric plasma is moved across the mask apertures. The plasma increases the surface energy where it is hitting the surface and so hydrophilizing the substrate through the mask apertures.
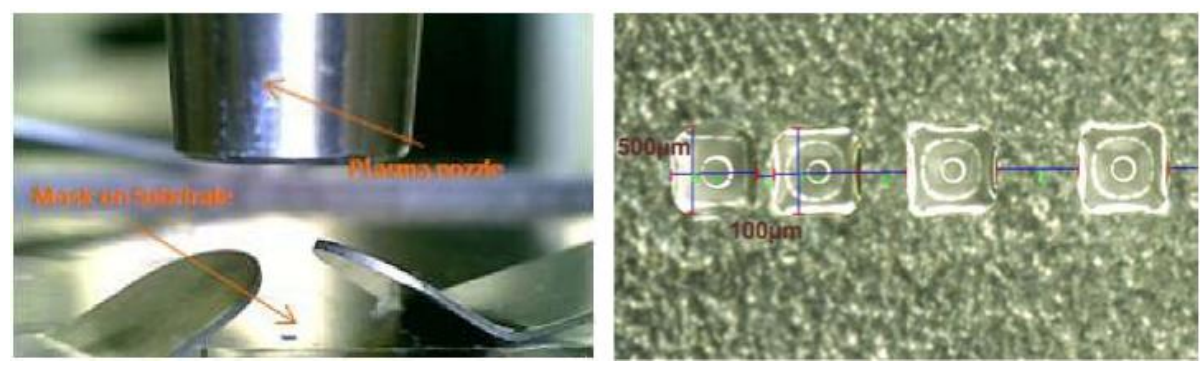

Fig. 3. Left: SMD-mask on polymer substrate and plasma nozzle above. Right: Droplets on functionalized surface with $500 \mu \mathrm{m}$ edge length and minimal spacing of $100 \mu \mathrm{m}$.

The results can be seen in figure 3 right, where droplets are positioned on the hydrophile areas to show the effect. Hydrophile areas with $500 \mu \mathrm{m}$ edge length have been realized with a sharp transition, which consequences the square shaped droplets. Also the realized $100 \mu \mathrm{m}$ gap between two droplets is an indicator for a sharp transfer of the shape of the mask apertures to the surface.

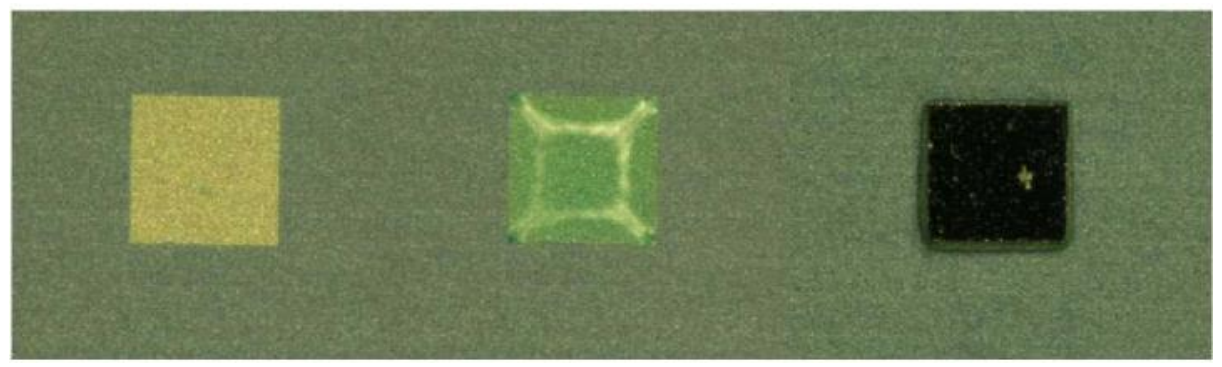

Fig. 4. Left: $500 \mu \mathrm{m}$ hydrophile square on an offset-printing plate. Centre: Inked water (for better visualization) on hydrophile area. Right: Self-assembled chip. 
Also good results have been achieved with the established technology for the manufacturing of offset-printing plate. A Teflon covered aluminum plate is structured with a laser to realize hydrophile and hydrophobic surfaces. In figure 4, the water droplet on the functionalized area shows even better characteristics as the atmospheric plasma functionalization. But the disadvantage of the adapted substrate material has to be mentioned. Structures with $200 \mu \mathrm{m}$ size can be realized easily with this method.

\subsection{Droplet Deposition}

Ultrapure water is deposited onto the hydrophile areas. The water evaporates residue-

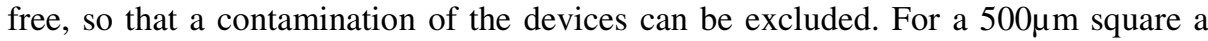
droplet with a diameter of approx. $400 \mu \mathrm{m}$ is needed. This is realized with an airpowered dispenser and a passive magnetic valve developed at Fraunhofer IPA, the so called IPA.VALVE [9]. The valve supports a precise volume control, which is an important parameter for a stable process. The valve can be miniaturized and integrated into capillaries as shown in figure 5. A parallel applying of droplets for a high throughput production can be achieved by an application specific arrangement of capillaries with integrated valves.
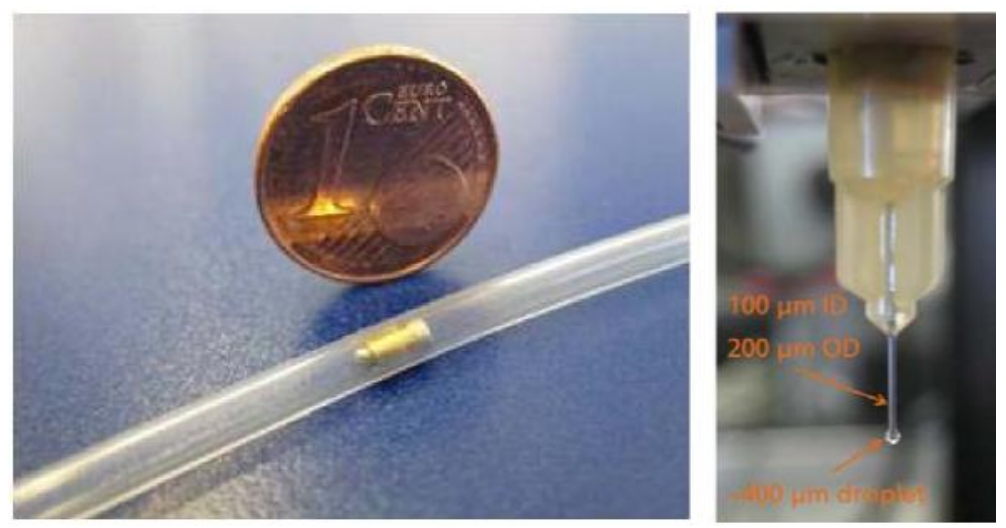

Fig. 5. Left: Miniaturized IPA.VALVE in a $1 \mathrm{~mm}$ tube. Right: $400 \mu \mathrm{m}$ droplet. $\varnothing$

\subsection{Device Placement}

The transfer of the device onto the droplet is realized without using a gripper. For that, the devices are arranged in a channel one after the other and moved in such a manner, that the last device protrudes from the magazine edge. The substrate with the droplet is moving underneath. As soon as the droplet, which is slightly higher as the substrate itself, is touching the device, it is grabbed by it and pulled out of the channel onto the droplet. The device gets positioned onto its target position according the functionalized surface due to the surface tension. 

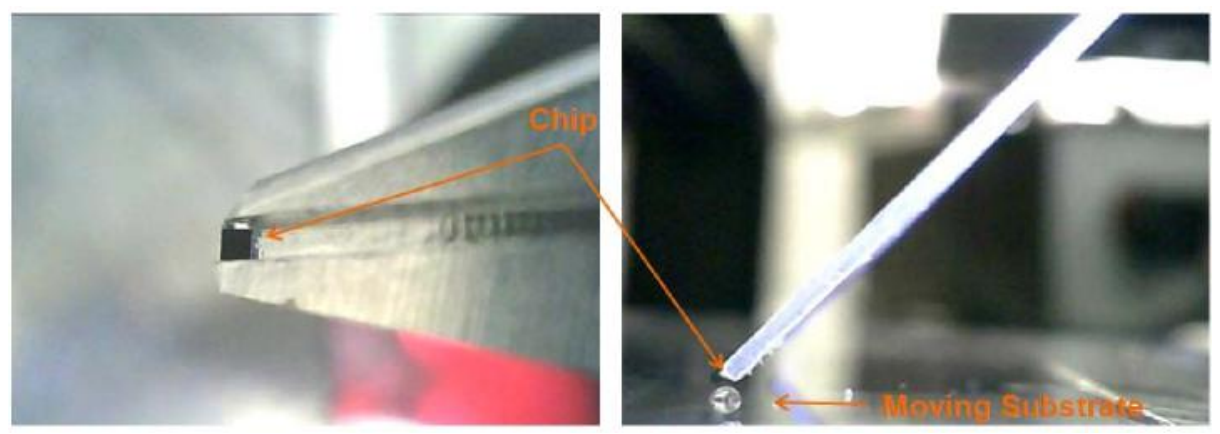

Fig. 6. Left: Top view onto the channel with a single chip protruding from the edge. Right: Side view with moving substrate and droplet underneath the chip.

\subsection{Integration into a Roll-to-Roll Process}

For a continuous process, as it is needed for a roll-to-roll process, the devices have to be deposited in an array onto a moving substrate. In figure 7 the moving substrate is described by a rotating cylinder. An offset printing plate is bended on the cylinder with functionalized squares of $500 \mu \mathrm{m}$ edge length. The droplets and devices are deposited in parallel with arranged capillaries and channels.

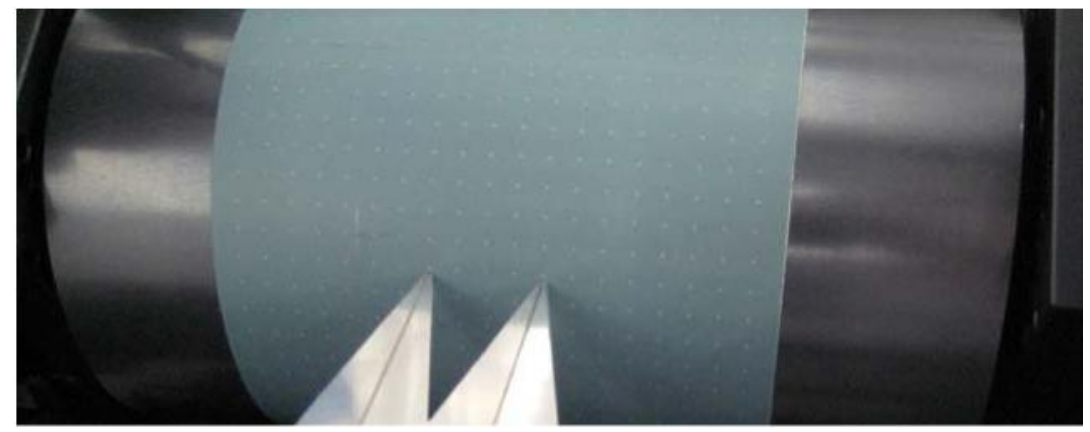

Fig. 7. Cylinder with functionalized surface and parallel deposition of devices

\section{Conclusion}

The processes for taking advantage of the self-assembly effect for the positioning of devices with an edge size of $500 \mu \mathrm{m}$ are described. The processes are adapted for a continuous roll-to-roll manufacturing. The requirements for a parallel positioning of devices within a roll-to-roll manufacturing are fulfilled with the above described method. Future work will be focused on the automation of the processes to meet the requirements of production equipments. 
Acknowledgments. The work leading to these results has received funding from the European Community's Seventh Framework Program under grant agreement $\mathrm{n}^{\circ} \mathrm{CPTP}$ 228686. www.light-rolls.eu

\section{References}

1. Boufercha, N., Sägebarth, J., Othman, N., Burgard, M., Schäfer, W., Sandmaier, H.: Selfassembly of Micro Components with Microfluidics. In: Proceedings of the International Workshop on Micro- and Nano- Technologies and Systems, Moskau, pp. 45-52 (2007)

2. Hecker, K.: Organic and Printed Electronics, 3rd edn. VDMA Verlag GmbH, Frankfurt am Main (2009)

3. Gourlay, J., et al.: Light-Rolls: High throughput manufacture for LED Lighting and Displays. Display Week 2010, SID International Symposium, Society for Information Display, 2010 - Digest of Technical Papers (2010) 20110104283

4. MINAM - Roadmaps, http : / /wWw. micronanomanufacturing . eu

5. Morris, C.J., Stauth, S.A., Parviz, B.A.: Self-Assembly for Microscale and Nanoscale Packaging: Steps Toward Self-Packaging. IEE Transactions on Advanced Packaging 28(4), 600-611 (2005)

6. Boufercha, N., Schäfer, W., Sägebarth, J., Sandmaier, H.: Influences of Nanostructured Interfaces on Microfluidics. In: NNT International Conference on Nanoprint \& Nanoimprint Technology (2007)

7. Fang, J., Böhringer, K.F.: Wafer-Level Packaging Based on Uniquely Orienting SelfAssembly (The DUO-SPASS Process). Journal of Microelectromechanical Systems 15(3) (June 2006)

8. Yeh, H.J., Smith, J.S.: Fluidic Slef-Assembly for the Integration of GaAs Light-Emitting Diodes on Si Substrates. IEEE Photonics Technology Letters 6(6) (June 1994)

9. Schlenker, D.: Schließventillösung für unterschiedlichste Anwendungen. adhäsion KLEBEN \& DICHTEN, 16-18 (November 2010) 